\title{
Fabrication of Visible-Light Sensitized ZnTe/ZnSe (Core/Shell) Type-II Quantum Dots
}

\author{
Misung Kim and Jiwon Bang ${ }^{\dagger}$ \\ Electronic Conversion Materials Division, Korea Institute of Ceramic Engineering and Technology, Jinju 52852, Korea \\ (Received August 24, 2018; Revised August 24, 2018; Accepted August 25, 2018)
}

\begin{abstract}
Colloidal semiconductor quantum dots (QDs), because of the novel optical and electrical properties that stem from their threedimensional confinement, have attracted great interest for their potential applications in such fields as bio-imaging, display, and opto-electronics. However, many semiconductors that can be exploited for QD applications contain toxic elements. Herein, we synthesized non-toxic ZnTe/ZnSe (core/shell) type-II QDs by pyrolysis method. Because of the unique type-II character of these QDs, their emission can range over an extended wavelength regime, showing photoluminescence (PL) from $450 \mathrm{~nm}$ to $580 \mathrm{~nm}$. By optimizing the ZnSe shell growth condition, resulting ZnTe/ZnSe type-II QDs shows PL quantum yield up to 25\% with $35 \mathrm{~nm}$ PL bandwidth. Using a simple two step cation exchange reaction, we also fabricated ZnTe/ZnSe type-II QDs with absorption extended over the whole visible region. The visible-light sensitized heavy metal free ZnTe/ZnSe type-II QDs can be relevant for opto-electronic applications such as displays, light emitting diodes, and bio-imaging probes.
\end{abstract}

Key words : ZnTe/ZnSe, Quantum dots, Optical properties, Nanocomposites

\section{Introduction}

$\mathrm{C}$ olloidal quantum dots (QDs) possess unique optical properties such as broad absorption with narrow emission features and tunabilily of size effect. ${ }^{1,2)}$ Furthermore, the photochemical stability and durability of quantum dots make them an ideal material for selective targeting and solution preparation because their surface can accommodate various molecular sieves. Many experiments are underway, and applications in development, in the display, ${ }^{3)}$ solar cell, ${ }^{4)}$ photo-detector, ${ }^{5)}$ as well as bio-sensor ${ }^{6)}$ and imaging-probe applications. ${ }^{7,8)}$ By controlling the size, any class of Cd QDs, in particular CdSe QDs, can be utilized to produce high luminescent light efficiency in a wide range of the visible light spectrum. These QDs also show a narrow photoluminescence (PL) features. Yet, their stability has attracted attention, making this a novel light emitting material. ${ }^{9,10)}$ However, the toxicity of $\mathrm{Cd}$ limits immediate application in various fields. Accordingly, A class of nontoxic QDs with containing In, in particular InP QDs, was studied. ${ }^{11,12)}$ Unfortunately, group III-V materials form more ionic bond characteristics, which makes it difficult to control the size in comparison to the cases of groups II-VI. In addition, the relatively wide band-width brings many restrictions in display and multi-functional molecular imaging. ${ }^{11,12)}$

The difference of energy level between heterogenous materials of group II-VI ZnTe and ZnSe in type-II ZnTe/

\footnotetext{
${ }^{\dagger}$ Corresponding author : Jiwon Bang

E-mail : jwbang@kicet.re.kr

Tel : +82-55-792-2677 Fax : +82-55-792-2492
}

ZnSe (core/shell) quantum dots have excited electrons at the conduction band of $\mathrm{ZnSe}$ and holes located at the valence electron band of ZnTe, forming a type-II heterostructure. ${ }^{13)}$ The effective band gap of this structure becomes narrower than that of $\mathrm{ZnSe}$ and $\mathrm{ZnTe}$, extended PL peak over visible spectral range. However, the conventional heterostructure of $\mathrm{ZnTe} / \mathrm{ZnSe}$ quantum dots poses a difficulty in mass production because the use of a highly reactive precursor such as diethylzinc is needed, which limits the control of the growth speed.

This paper attempts to control various precursors to produce a composite of ZnTe/ZnSe (core/shell) type-II QDs that has bright PL characteristics in the visible light spectrum and $\mathrm{ZnTe} / \mathrm{ZnSe}$ quantum dots that have a narrow PL bandwidth. Furthermore, we show a composite product of $\mathrm{ZnTe} /$ ZnSe (core/shell) type-II quantum dots that absorbs whole visible spectral range via cation exchange reaction.

\section{Experimental Procedure}

\subsection{Reagent}

The following reagents are used in the experiment: diethylzinc solution (1.0 M in hexanes, Aldrich), hexadecylamine (HDA, 98\%, Aldrich), 1-octadecene (ODE, 90\%, Aldrich), tellurium powder (Te, 99.997\%, Aldrich), selenium powder (Se, 99.99\%, Aldrich), zinc acetate $\left(\mathrm{Zn}(\mathrm{Ac})_{2}, 99.99 \%\right.$, Aldrich), zinc chloride ( $\mathrm{ZnCl}_{2}, 99.999 \%$, Aldrich), oleic acid (OA, 90\%, Sigma-Aldrich), oleylamine (70\%, Aldrich), trioctylphosphine (TOP, 97\%, Strem), cadmium acetate dihydrate (98\%, Sigma-Aldrich), tetradecyl phosphonic acid (TDPA, 97\%, Aldrich), and tetrakis (acetonitrile) copper (I) hexafluoro- 
phosphate $\left(\left[\mathrm{Cu}\left(\mathrm{CH}_{3} \mathrm{CN}_{4}\right] \mathrm{PF}_{6}, 97 \%\right.\right.$, Aldrich).

\section{2. $Z n T e$ core $Q D$}

TOP-Te precursor was prepared in a glove box in which Te was melted to $0.5 \mathrm{M}$ in TOP. Then, $2.5 \mathrm{~g}$ of HDA and 7 $\mathrm{ml}$ of ODE were heat treated for an hour in vacuum. The environment was then fixed with nitrogen. After heat treatment, at up to $150^{\circ} \mathrm{C}$, a mixed solution of diethylzinc and TOP-Te was quickly injected and stirred with heat for 20 minutes at $250^{\circ} \mathrm{C}$.

\subsection{ZnTe/ZnSe core/shell QDs}

2.3.1. Composite production with use of diethylzinc precursor

The work used the SILAR ${ }^{14)}$ coating approach method as the basis of ZnTe/ZnSe core/shell QDs. First, for the preparation of TOP-Se precursor, Se was melted to $0.5 \mathrm{M}$ in TOP inside a glove box. Then, ZnTe QDs prepared in 2.2 were combined with diethylzinc precursor and TOP-Se precursor, with increase of temperature from $170^{\circ} \mathrm{C}$ to $300^{\circ} \mathrm{C}$. The temperature was increased with mixed insertion at every shell level and ZnSe shell levels were laminated. Finally, a large volume of ethanol was introduced to quantum dot solution to produce a precipitate, which was then dispersed with hexane later.

2.3.2. Composite production with use of Zinc oleate precursor

Zinc oleate precursor was produced using $\mathrm{Zn}(\mathrm{Ac})_{2} \mathrm{mmol}$, OA mmol, and ODE ml. First, $\mathrm{Zn}(\mathrm{Ac})_{2}$ mmol, OA mmol and ODE $\mathrm{ml}$ were heated for $1 \mathrm{~h}$ in a vacuum incubator at $110^{\circ} \mathrm{C}$. Then, solutions were left in nitrogen environment under $200^{\circ} \mathrm{C}$ for an hour for reaction to take place. After that, injection similar to that shown in 2.3 (1) was done, but instead of diethylzinc, zinc oleate precursor was injected.

\subsubsection{Composite production with use of Zinc chloride pre-} cursor

Zinc chloride precursor was produced using $\mathrm{ZnCl}_{2} 4 \mathrm{mmol}$, Oleylamine $7.2 \mathrm{ml}$ and ODE $12.8 \mathrm{ml}$. These solutions were put under $110^{\circ} \mathrm{C}$ of vacuum for an hour with heat exchange. After that, solutions were introduced to nitrogen environment for an hour under $200^{\circ} \mathrm{C}$ to prepare zinc chloride precursor. As in 2.3 (1), zinc chloride precursor was injected instead of diethylzinc.

\subsubsection{Cation exchange}

- Synthesis of CdTe/CdSe (core/shell) QDs

cadmium acetate dihydrate $0.4 \mathrm{mmol}$, TDPA $0.9 \mathrm{mmol}$ and ODE $4 \mathrm{ml}$ were heat stirred under $110^{\circ} \mathrm{C}$ in vacuum for an hour. Then, the environment was changed to nitrogen and the temperature was increased to room temperature, at which point Oleylamine $2 \mathrm{ml}$ and ODE $20 \mathrm{ml}$ were injected. Solutions were again heated to $300^{\circ} \mathrm{C}$ to prepare $\mathrm{Cd}$ precursor. TOP-Te $0.2 \mathrm{mmol}$ was injected into CD precursor to produce CdTe nuclear quantum dot composite. Then, a

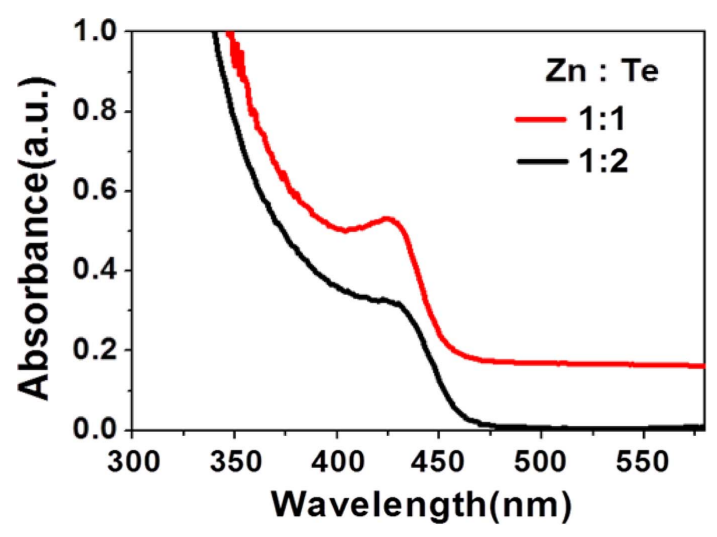

Fig. 1. Absorption spectra of ZnTe QDs with $1: 1$ (black) and 1 : 2 (red) molar ratio of $\mathrm{Zn}$ and Te.

large volume of ethanol was introduced to create quantum dot precipitate. After the precipitate was produced, a mix of ODE $15 \mathrm{ml}$ and oleylamine $5 \mathrm{ml}$ was heat stirred under $110^{\circ} \mathrm{C}$ in vacuum for an hour. After injection, CdTe/CdSe with a CdSe shell layer grown on the surface of CdTe nuclei was synthesized in an incubator under heat treatment.

\section{- $\mathrm{Cu}_{2} \mathrm{Te} / \mathrm{Cu}_{2} \mathrm{Se}$ Cation exchange reaction}

$\mathrm{Cu}$ precursor was prepared using $\left.\mathrm{Cu}\left(\mathrm{CH}_{3} \mathrm{CN}\right)_{4}\right] \mathrm{PF}_{6}$ in $3 \mathrm{ml}$ of methanol. The composite of $\mathrm{CdTe} / \mathrm{CdSe}$ from above was dispersed using toluene and injected into $\mathrm{Cu}$ precursor. Mixtures were stirred at room temperature for 5 minutes for cation exchange. The exchanged nano crystals were purified using methanol and then introduced into TOP.

\section{- ZnTe/ZnSe cation exchange reaction}

$\mathrm{ZnCl}_{2} 4 \mathrm{mmol}$, ODE $8 \mathrm{ml}$ and olelyamine $6 \mathrm{ml}$ were heat treated in a vacuum for an hour at $110^{\circ} \mathrm{C}$. Solutions were again heated to $250^{\circ} \mathrm{C}$ after moving to a nitrogen environment. Then, mixture of $\mathrm{Cu}_{2} \mathrm{Te} / \mathrm{Cu}_{2} \mathrm{Se}$ from above was injected and heat treated from 5 to 20 minutes to allow cation exchange to take place.

\section{Results and Discussion}

A composite of $\mathrm{ZnTe}$ core QDs can be made by quickly injecting high temperature ODE solvent that contains hexadecylamine surfactant and TOP-Te precursor. The highly reactive diethylzinc will induce the formation of a nucleus and its growth in a reactive vessel under high temperature will produce a uniformal size of $\mathrm{ZnTe}$ core quantum dots. The $\mathrm{Zn}$ :Te ratio that allows for uniformal size production of ZnTe quantum dots is $1: 1$ and not $1: 2$ (Fig. 1). The use of a $1: 1$ ratio of $\mathrm{Zn}$ :Te quantum dot showed a sharp 1st exciton absorption peak. A ZnTe composite prepared in $1: 1$ ratio of $\mathrm{Zn}$ and Te precursor shows an absorption peak at about 430 $\mathrm{nm}$, but no fluorescence. This is due to surface defects of ZnTe QDs, which suppress radiation emission.

ZnTe/ZnSe (core/shell) QDs are made using SILAR (Successive Ionic Layer Adsorption and Reaction) with $\mathrm{Zn}$ and 
Se precursors in a reactive vessel to grow ZnSe shell layers. TOP-Se is used as the Se precursor. Also, Zn precursor is made using either diethylzinc, zinc oleate or zinc chloride to induce ZnSe shell layer growth. Fig. 2 shows the optical characteristics of different QDs that use $\mathrm{Zn}$ precursor in the reaction.

As can be seen in Fig. 2(a), the red shifted absorption profile of the $\mathrm{ZnTe}$ core QDs is barely visible during diethylzinc and TOP-Se cross injection. ZnSe shell layer growth is not observed either. It is expected that diethylzinc is oxidized before it is injected into the TOP-Se reactive vessel, producing a layer of oxidation during the SILAR coating process instead of leading to $\mathrm{ZnSe}$ shell layer growth on the surface of ZnTe. Furthermore, after injecting $\mathrm{Zn}$ and Se precursors, a weak yet broad emission is observed near $\sim 600 \mathrm{~nm}$ of the wave range. We believe this trap emission is due to a formation of an oxidative layer on the surface of ZnTe quantum dots. ${ }^{15)}$ Although the diethylzinc precursor is reactive, Fig. 2(a) shows that it is not an adequate precursor for $\mathrm{ZnSe}$ shell growth when using the SILAR coating method.

When using a $\mathrm{Zn}$ precursor of zinc ion coordinated to a carboxylate group, as in zinc-oleate (Fig. 2(b)) or zinc chloride (Fig. 2(c)), progressive red shifted absoption and PL spectra are observed during the continuous SILAR coating method. This is due to the thickening of the ZnSe shell, which lowers the energy in the conduction band of $\mathrm{ZnSe}$ and narrows the effective band gap of the $\mathrm{ZnTe} / \mathrm{ZnSe}$ (core/shell)
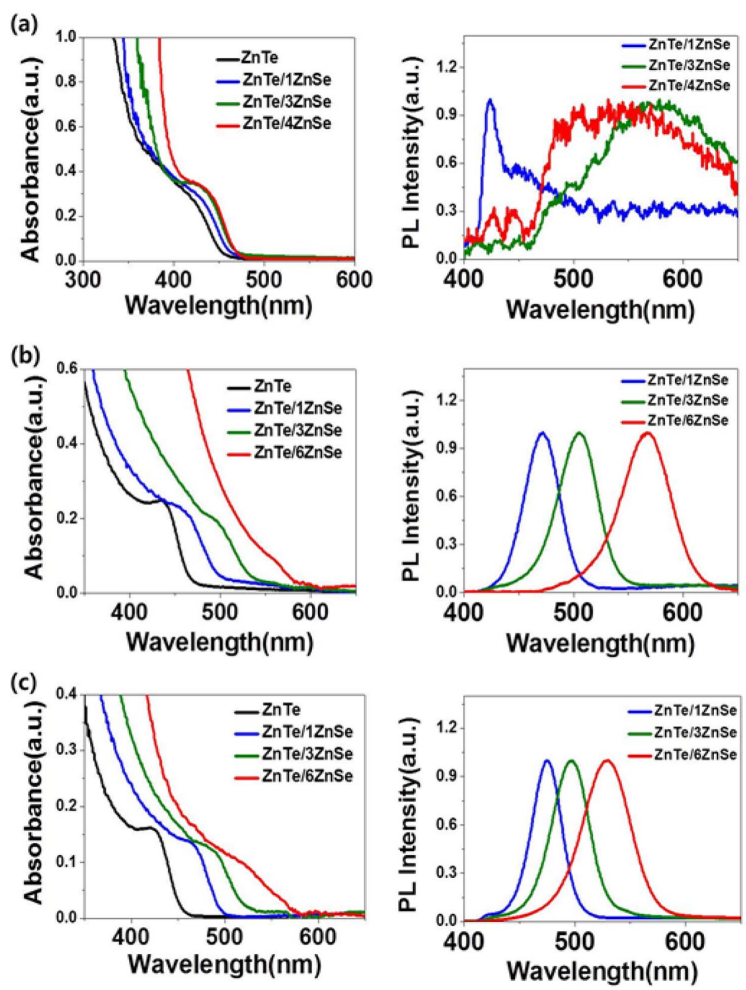

Fig. 2. Absorption (left panel) and photoluminescence spectra (right panel) of ZnTe core and ZnTe/ZnSe (core/ shell) QDs with (a) diethylzinc, (b) zinc-oleate, and (c) zinc chloride as zinc precursor for ZnSe shell growth.
QDs.

Furthermore, as the thickness of the ZnSe shell layer increases, the spatial overlap between electron and hole decreases, and the 1st exciton absorption peak gradually becomes featureless. ${ }^{13)}$ Not only the red-shifted PL feature as the ZnSe shell layer of the ZnTe/ZnSe (core/shell) QDs grows, but the $\mathrm{ZnSe}$ layers allow passivation of the surface of ZnTe QDs. Furthermore, overlap integral between photoexcited charge carrier and surface trapped carrier is reduced, resulting in improved fluorescent efficiency and stability. ZnTe QDs were coated with SILAR coating six times to produce a composite of $\mathrm{ZnTe} / \mathrm{ZnSe}$ (core/shell) QDs. Using the Zn-oleate precursor to coat the ZnSe shell produced $12 \%$ of PL efficiency, showing a PL band-width at 40 $\mathrm{nm}$. Using $\mathrm{ZnCl}_{2}$ to coat the $\mathrm{ZnSe}$ shell produced $25 \% \mathrm{PL}$ efficiency with PL band-width at $35 \mathrm{~nm}$. This PL efficiency is an improvement compared to previous studies, ${ }^{13)}$ where ZnTe QDs were introduced to diethylzinc and TOP-Se to increase ZnSe shell layers for the final product of $\mathrm{ZnTe} /$ ZnSe QDs. The use of a stable $\mathrm{Zn}$ precursor, along with SILAR coating of the ZnTe nucleus, allowed for uniform shell growth of $\mathrm{ZnSe}{ }^{14,16)}$ Also, using $\mathrm{ZnCl}_{2}$ instead of $\mathrm{Zn}$ oleate improved the PL efficiency because chloride ion allows for passivation on the surfaces of groups II-VI nanoparticles, eliminating trap sites and thereby increasing the stability. ${ }^{17,18)}$ Therefore, it is believed that $\mathrm{ZnCl}_{2}$ precursor allows not only for ZnSe shell growth but also for surface passivation, which increases PL efficiency.

In order to accurately represent color in the latest display models, the application of non-toxic quantum dots in the visible light spectrum is becoming crucial and requires a material with very narrow PL band-width. ${ }^{19)}$ This paper attempted to compare the characteristics of a new composite of $\mathrm{ZnTe} / \mathrm{ZnSe}$ (core/shell) QDs fluorescent with those of a composite of nontoxic InP/ZnSe (core/shell). ${ }^{20,21)}$ First, InP/ ZnSe has limited display applications because it is in groups III-V, in which quantum dots have a wide PL bandwidth. ${ }^{20,21)}$ As can be seen in Fig. 3, green emitting InP/ZnSe QDs have a band-width at $52 \mathrm{~nm}$. However, the composite produced in this study, ZnTe/ZnSe (core/shell) QDs, has a narrower PL band-width in the same PL peak region. It was observed that, in comparison to InP based composite, the $\mathrm{ZnTe} / \mathrm{ZnSe}$ (core/shell) composite can be used to better represent true colors.
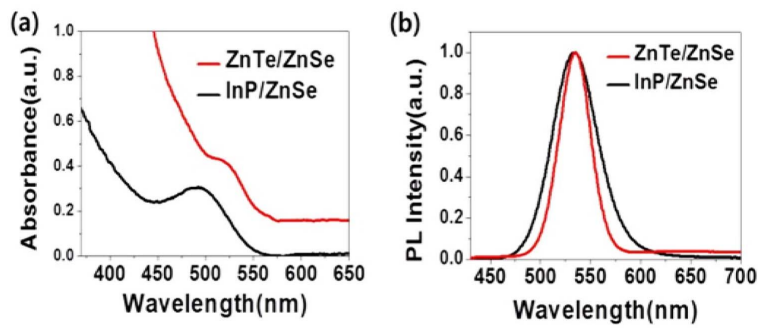

Fig. 3. Comparison of (a) absorption and (b) photoluminescence spectra of green emitting InP/ZnSe (core/shell) QDs (black) and ZnTe/ZnSe (core/shell) QDs (red). 
(a)

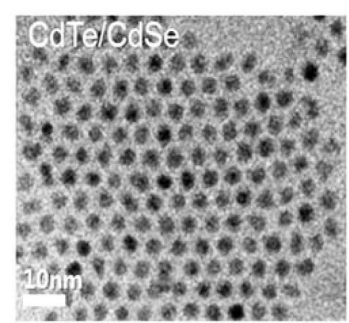

(c)

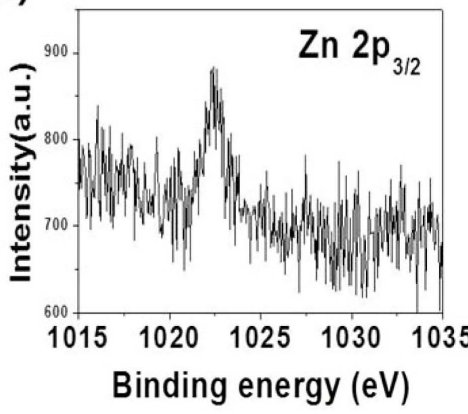

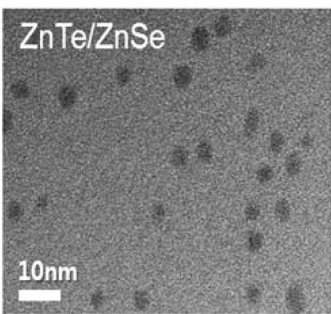

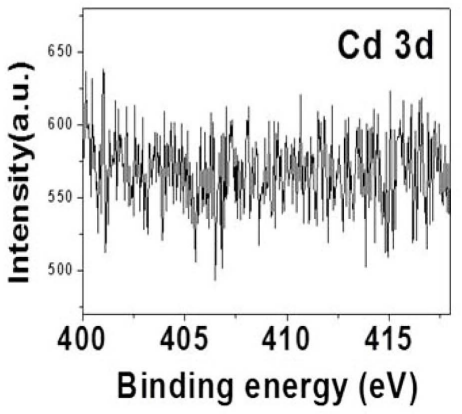

(b)
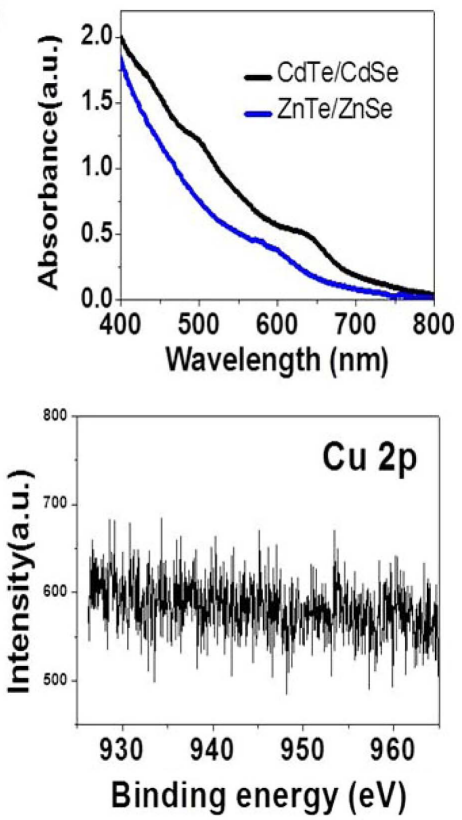

Fig. 4. (a) TEM image and (b) optical absorption spectra of starting CdTe/CdSe (core/shell) QDs and corresponding ZnTe/ZnSe (core/shell) QDs obtained via cation exchange reaction. (c) XPS spectra of corresponding ZnTe/ZnSe (core/shell) QDs

Figure 2 shows the $\mathrm{ZnTe} / \mathrm{ZnSe}$ (core/shell) quantum dot effective band gap to have more extend to red color as the ZnSe shell layers thickens. However, there are limitations in absorbing/emitting in ranges beyond than $600 \mathrm{~nm}$. It is conjectured that this phenomenon occurs because perhaps $\mathrm{ZnTe}$ and ZnSe have a large lattice mismatch (7.1\%) that limits ZnSe shell layer growth. Producing a ZnTe/ZnSe (core/shell) QDs that responds to the red emitting was necessary. A $6.0 \mathrm{~nm}$ sized type-II CdTe/CdSe (core/shell) quantum dot ${ }^{22)}$ was utilized and underwent sequential cation exchange of $\mathrm{Cd}^{2+} \rightarrow \mathrm{Cu}^{+} / \mathrm{Cu}^{+} \rightarrow \mathrm{Zn}^{2+}{ }^{23)}$ Fig. 4 shows the large size of the ZnTe/ZnSe (core/shell) QD product. Figs. 4(a) represents TEM images of starting CdTe/CdSe (core/ shell) QDs and the corresponding ZnTe/ZnSe (core/shell) QDs obtained via cation exchange. The CdTe/CdSe (core/ shell) QDs had an average size of $6.0 \mathrm{~nm}$, but the size decreased to an average of $5.6 \mathrm{~nm}$ after the cation exchange reaction. The QDs did keep their spherical shape. The average particle size decreased by $6.7 \%$ because the lattice constant of Zn-chalcogenide is smaller than that of $\mathrm{Cd}$ chalcogenide lattice when the exchange from $\mathrm{Cd}$ to $\mathrm{Zn}$ shrinks the lattice ('CdTe with ZnTe' and 'CdSe with ZnSe' lattice mismatches are $5.9 \%$ and $6.7 \%$, respectively). Absorption onset can be observed at $680 \mathrm{~nm}$ for the $6.0 \mathrm{~nm}$ size CdTe/CdSe (core/shell) quantum dots. On the other hand, the $5.6 \mathrm{~nm}$ sized ZnTe/ZnSe (nucleus/shell) quantum dots after cation exchange show absorption onset at $620 \mathrm{~nm}$ (Fig. 4(b)). Zn-chalcogenize has a wider band gap than that of Cd-chalcogenide, and thereby has bluish absorption. Fig. 4(c) shows XPS analysis of $\mathrm{Cd}$ and $\mathrm{Cu}$ ions are replaced by $\mathrm{Zn}$ ions after cation exchange of ZnTe/ZnSe (core/shell) QDs. It was confirmed that synthesizing $\mathrm{ZnTe} / \mathrm{ZnSe}$ (nucleus/ shell) quantum dots that respond to over $600 \mathrm{~nm}$ long waves was possible by performing cation exchange to form similarsized CdTe/CdSe (core/shell) QDs.

\section{Conclusions}

In this work, we synthesized a composite of $\mathrm{ZnTe} / \mathrm{ZnSe}$ (core/shell) type-II QDs that absorbs and emits visible light. ZnTe core QDs were synthesized via high temperature thermal decomposition to maximize the ZnSe shell layer and create visible light-emitting $\mathrm{ZnTe} / \mathrm{ZnSe}$ (core/shell) type-II quantum dots that have a maximum 25\% PL efficiency with half-width of $35 \mathrm{~nm}$. Through further study, ZnTe/ZnSe (core/shell) type-II QDs that can absorb the entire visible light spectrum, using large $\mathrm{CdTe} / \mathrm{CdSe}$ (core/shell) type-II QDs with two-step cation exchange reaction, were obtained. These environmentally friendly quantum dots that absorb and emit visible light are expected to be utilized in the field of highly efficient photovoltaic and in high definition display materials.

\section{Acknowledgments}

This research was supported by the Policy Research Project of the Korea Institute of Ceramic Engineering and Technology (KPP_18005).

\section{REFERENCES}

1. M. B. Jr, M. Moronne, P. Gin, S. Weiss, and A. P. Alivisatos, "Semiconductor Nanocrystals as Fluorescent Biological Labels," Science, 281 [5385] 2013-16 (1998). 
2. J. Lee, V. C. Sundar, J. R. Heine, M. G. Bawendi, and K. F. Jensen, "Full Color Emission from II-VI Semiconductor Quantum Dot-Polymer Composites," Adv. Mater., 12 [15] 1102-05 (2000).

3. Y. Jiang, S. Y. Cho, and M. Shim, "Light-Emitting Diodes of Colloidal Quantum Dots and Nanorod Heterostructures for Future Emissive Displays," J. Mater. Chem. C, 6 [11] 2618-34 (2018).

4. R. Liu, B. P. Bloom, D. H. Waldeck, P. Zhang, and D. N. Beratan, "Improving Solar Cell Performance Using Quantum Dot Triad Charge-Separation Engines," J. Phys. Chem. C, 122 [11] 5924-34 (2018).

5. K. S. Cho, K. Heo, C. W. Baik, J. Y. Choi, H. Jeong, S. Hwang, and S. Y. Lee, "Color-Selective Photodetection from Intermediate Colloidal Quantum Dots Buried in Amorphous-Oxide Semiconductors," Nat. Commun., 8 [1] 840 (2017)

6. P. T. Snee, R. C. Somers, G. Nair, J. P. Zimmer, M. G. Bawendi, and D. G. Nocera, "A Ratiometric CdSe/ZnS Nanocrystal pH Sensor," J. Am. Chem. Soc., 128 [41] 13320-21 (2006).

7. T. T. Xuan, J. Q. Liu, C. Y. Yu, R. J. Xie, and H. L. Li, "Facile Synthesis of Cadmium-Free Zn-In-S:Ag/ZnS Nanocrystals for Bio-Imaging," Sci. Rep., 624459 (2016).

8. X. Michalet, F. F. Pinaud, L. A. Bentolila, J. M. Tsay, S. Doose, J. J. Li, G. Sundaresan, A. M. Wu, S. S. Gambhir, and S. Weiss, "Quantum Dots for Live Cells, in Vivo Imaging, and Diagnostics," Science, 307 [5709] 538-44 (2005).

9. M. Danek, K. F. Jensen, C. B. Murray, and M. G. Bawendi, "Synthesis of Luminescent Thin-Film CdSe/ZnSe Quantum Dot Composites Using CdSe Quantum Dots Passivated with an Overlayer of ZnSe," Chem. Mater., 8 [1] 17380 (1996)

10. B. Xing, W. Li, X. Wang, H. Dou, L. Wang, K. Sun, X. He, J. Han, H. Xiao, J. Miao, and Y. Li, "Highly-Fluorescent Alloyed Quantum Dots of $\mathrm{CdSe}_{1-\mathrm{x}} \mathrm{Te}_{\mathrm{x}}$ Synthesized in Paraffin Liquid: Gradient Structure and Promising BioApplication," J. Mater. Chem., 20 [27] 5664-74 (2010.)

11. E. Bang, Y. Choi, J. Cho, Y. H. Suh, H. W. Ban, J. S. Son, and J. Park, "Large-Scale Synthesis of Highly Luminescent InP@ZnS Quantum Dots Using Elemental Phosphorus Precursor," Chem. Mater., 29 [10] 4236-43 (2017).

12. S. Tamang, C. Lincheneau, Y. Hermans, S. Jeong, and P. Reiss, "Chemistry of InP Nanocrystal Syntheses," Chem. Mater., 28 [8] 2491-506 (2016).

13. J. Bang, J. Park, J. H. Lee, N. Won, J. Nam, J. Lim, B. Y. Chang, H. J. Lee, B. Chon, J. Shin, J. B. Park, J. H. Choi, K. Cho, S. M. Park, T. Joo, and S. Kim, "ZnTe/ZnSe (Core/ Shell) Type-II Quantum Dots: their Optical and Photovoltaic Properties," Chem. Mater., 22 [1] 233-40 (2010).
14. Y. Chen, J. Vela, H. Htoon, J. L. Casson, D. J. Werder, D. A. Bussian, V. I. Klimov and J. A. Hollingsworth, "Giant" Multishell CdSe Nanocrystal Quantum Dots with Suppressed Blinking," J. Am. Chem. Soc., 130 [15] 5026-27 (2008).

15. W. H. Zhang, J. L. Shi, L. Z. Wang, and D. S. Yan, "Preparation and Characterization of $\mathrm{ZnO}$ Clusters inside Mesoporous Silica," Chem. Mater., 12 [5] 1408-13 (2000).

16. C. Lincheneau, M. Amelia, M. Oszajca, A. Boccia, F. D'Orazi, M. Madrigale, R. Zanoni, R. Mazzaro, L. Ortolani, V. Morandi, S. Silvi, K. Szaciłowski, and A. Credi, "Synthesis and Properties of ZnTe and ZnTe/ZnS Core/ Shell Semiconductor Nanocrystals," J. Mater. Chem. C, 2 [6] 2877-86 (2014).

17. J. Y. Woo, J. H. Ko, J. H. Song, K. Kim, H. Choi, Y. H. Kim, D. C. Lee, and S. Jeong, "Ultrastable PbSe Nanocrystal Quantum Dots via in Situ Formation of Atomically Thin Halide Adlayers on PbSe(100)," J. Am. Chem. Soc., 136 [25] 8883-86 (2014).

18. R. C. Page, D. Espinobarro-Velazquez, M. A. Leontiadou, C. Smith, E. A. Lewis, S. J. Haigh, C. Li, H. Radtke, A. Pengpad, F. Bondino, E. Magnano, I. Pis, W. R. Flavell, P. O'Brien, and D. J. Binks, "Near-Unity Quantum Yields from Chloride Treated CdTe Colloidal Quantum Dots," Small, 11 [13] 1548-54 (2015).

19. Y. C. Shih and F. G. Shi, "Quantum Dot Based Enhancement or Elimination of Color Filters for Liquid Crystal Display," IEEE J. Sel. Top. Quantum Electron., 23 [5] 1-4 (2017).

20. W. Shen, H. Tang, X. Yang, Z. Cao, T. Cheng, X. Wang, Z. Tan, J. You, and Z. Deng, "Synthesis of Highly Fluorescent InP/ZnS Small-Core/Thick-Shell Tetrahedral-Shaped Quantum Dots for Blue Light-Emitting Diodes," J. Mater. Chem. C, 5 [32] 8243-49 (2017).

21. J. Zhang; J. Wang, T. Yan, Y. Peng, D. Xu, and D. Deng, "InP/ZnSe/ZnS Quantum Dots with Strong Dual Emissions: Visible Excitonic Emission and Near-Infrared Surface Defect Emission and their Application in in vitro and in vivo Bioimaging," J. Mater. Chem. B, 5 [41] 8152-60 (2017)

22. J. Bang, B. Chon, N. Won, J. Nam, T. Joo, and S. Kim, "Spectral Switching of Type-II Quantum Dots by Charging," J. Phys. Chem. C, 113 [16] 6320-23 (2009).

23. H. Li, R. Brescia, R. Krahne, G. Bertoni, M. J. P. Alcocer, C. D'Andrea, F. Scotognella, F. Tassone, M. Zanella, M. De Giorgi, and L. Manna, "Blue-UV-Emitting ZnSe(Dot)/ ZnS(Rod) Core/Shell Nanocrystals Prepared from CdSe/ CdS Nanocrystals by Sequential Cation Exchange," ACS Nano, 6 [2] 1637-47 (2012). 